\title{
Elaboration of Nanoparticles Containing Indomethacin: Argan Oil for Transdermal Local and Cosmetic Application
}

\author{
Waisudin Badri, ${ }^{1}$ Karim Miladi, ${ }^{1}$ Rkia Eddabra, ${ }^{2}$ Hatem Fessi, ${ }^{1}$ and Abdelhamid Elaissari ${ }^{1}$ \\ ${ }^{1}$ University of Lyon 1, Villeurbanne, CNRS, UMR 5007, LAGEP-CPE, 43 boulevard 11 Novembre 1918, 69622 Villeurbanne, France \\ ${ }^{2} E c o l e$ Polytechnique de l'Université Internationale d'Agadir, Unité RDI en Génie Industriel et Génie des Procédés, Technopole d'Agadir, \\ BP 8143, Agadir, Morocco
}

Correspondence should be addressed to Abdelhamid Elaissari; elaissari@lagep.univ-lyon1.fr

Received 13 November 2014; Revised 18 February 2015; Accepted 18 February 2015

Academic Editor: Zhongkui Hong

Copyright (C) 2015 Waisudin Badri et al. This is an open access article distributed under the Creative Commons Attribution License, which permits unrestricted use, distribution, and reproduction in any medium, provided the original work is properly cited.

\begin{abstract}
The objective of this work is the preparation of nanocapsules which are intended for the treatment of rheumatoid arthritis (RA). We use Argan oil as vehicle of hydrophobic drugs such as indomethacin. Nanoprecipitation technique was used like a method for preparation of nanocapsules, where polycaprolactone, acetone, and Argan oil are used as polymer, solvent, and carrier, respectively. Primarily, investigation of Argan oil amount addition was performed; then systematic study to optimize the formulation was done. Nanocapsules containing indomethacin (loaded nanocapsules) and others without indomethacin (blank nanocapsules) were characterized by studying particles size, zeta potential, and encapsulation efficiency. It is shown that particle size ranged between $290 \mathrm{~nm}$ and $350 \mathrm{~nm}$. The zeta potential values were negative (from -40 up to $-50 \mathrm{mV}$ ) while encapsulation efficiency ranged between 65 and $75 \%$.
\end{abstract}

\section{Introduction}

The drug delivery systems design, which boosts drug efficiency by increasing the drug solubility and bioavailability, is the main point for pharmaceutical research. Moreover, by increasing the bioavailability or choosing alternative administration route, utilization of biomedical nanotechnology administration route associated undesirable effects (gastrointestinal tract disorders) might be reduced. The polymer based vehicles with nanometric size are capable utensils to (a) protect, (b) target, and (c) enhance intrinsic characteristics of the encapsulated drugs [1-3]. In transdermal drug delivery, the technology of nanoencapsulation signifies a smart approach to attain biodistribution of drug. The reason of this capacity is related to the good control of release, avoiding drug degradation, enhancing penetration efficiency of active molecule into the skin, and preventing toxic effects [4]. Commonly, six classical techniques for the preparation of nanocapsules such as nanoprecipitation, emulsion-diffusion, double emulsification, emulsion-coacervation, polymer-coating, and layer-bylayer are employed [5].
The choice of the encapsulation method is related to the required application, the nature of the active molecule and that of the polymer, and their physicochemical properties. The nature of vehicles plays role in selection of appropriate method for drug encapsulation [6].

Rheumatoid arthritis (RA) is one of the prevalent chronic health conditions. However, despite new development in medical treatment, RA therapy still represents an unmet medical need because of safety and efficacy worries with presently advised medications. Consecutively, there is a crucial need to develop and test new drugs for RA, which target in a selective approach the inflamed joints, thus alleviating damage to healthy tissues $[7,8]$.

Nonsteroidal anti-inflammatory drugs (NSAIDs) have considerable anti-inflammatory properties. NSAIDs through oral administration are very useful, but their employment in the clinic is limited because of their undesirable effects like irritation and ulceration of the digestive system mucosa.

On one hand the usage of these medications via the dermal route can remove these drawbacks of oral route administration and on the other hand nanomedicine could 
play significant role in improving long-term outcome of RA patients. Moreover, nanomedicine offers selective control of the inflammatory process in affected joints. To this end, the conception of drug nanocarrier for antirheumatic drugs is too attractive.

NSAIDs are widely advised for the RA treatment. Certain NSAIDs especially those that have selective antiinflammatory properties through the blockage of COX-II have shown the myocardial infarction, stroke, and cardiovascular death. It has been found that cardiovascular risks of NSAIDs are considerably different from each other. It should be kept in mind that long-term use of NSAIDs (nonselective COX-I and COX-II inhibitors) often induces the digestive system bleeding and stomach ulcers.

Nanotechnology enhances drug penetration to skin, decreases number of drug intakes, decreases drug dose to be taken and side effects, and sustains anti-inflammatory effect for a long time.

Indomethacin is a prime drug used for the treatment of RA, gout, and collagen disease. It is a potent inhibitor of cyclooxygenases, reducing prostaglandin synthesis, relieving pain, and reducing fever in febrile patients [9].

Furthermore, Argan oil is also included in this formulation because of two reasons, firstly to make soluble the indomethacin and create the cosmetic effect and antiinflammatory effect of the Argan oil. To our information, no work based on Argan oil in the preparation of polycaprolactone (PCL) nanoparticles containing active ingredient such as indomethacin (NSAIDs) to prepare the dispersion for derma-therapy in RA was reported. So the usage of PCL, Argan oil, and indomethacin for dermal-transdermal local and cosmetic application makes this work original. Moreover, composition of Argan oil including phytosterol, tocopherols, carotenes, polyphenols, and squalene leads to smart antiaging, moisturizing, and antioxidant effects; see Table 3 [10].

To date, treatment through human skin is significant and might diminish or even prevent the mucosal irritation. This purpose might be attained by formulating indomethacinloaded nanoparticles which offer several advantages including a high concentration of active molecule at the site of the lesion with a simultaneous very low concentration in the blood circulation, a continuous drug delivery, and a minimization of local damage to the gastrointestinal tract (GIT) mucosa.

In this investigation the Argan oil was added to the nanocapsules preparation through the nanoprecipitation technique. This is too appropriate method for the encapsulation of hydrophobic molecules. It is easy and simple approach of encapsulation.

The aim of the present work is in general the development of Argan oil based nanocapsules as carrier of hydrophobic molecules like indomethacin employed such as model. Firstly, a systematic study of nanoparticles formulation without indomethacin was performed. Studied parameters include materials related parameters (polymer concentration (polycaprolactone), Argan oil existence, Argan oil concentration, organic/aqueous phase ratio, stabilizer nature, and concentration) and operating conditions related parameters (like stirring speed, organic phase addition speed, and temperature). Effects on colloidal properties (i.e., particles size, zeta potential, stability, and morphology) were assessed. Secondly, the encapsulation of indomethacin, its characterization, stability study, and reproducibility test had been performed. Lococo et al. and Valentina et al. have, respectively, prepared the Argan oil nanocapsules containing diclofenac and naproxen as anti-inflammatory drug, as model drugs.

\section{Materials and Methods}

2.1. Materials. Acetone, polycaprolactone (PCL) $(\mathrm{Mw}=$ $14,000 \mathrm{~g} / \mathrm{mol})$, and polyvinyl alcohol (PVA) $(\mathrm{Mw}=$ 31,000 g/mol) were obtained from Sigma-Aldrich, Germany. Deionised water, Zetasizer (Malvern, UK), span 20, and indomethacin were supplied by VWR and Argan oil was provided by Universiapolis, Agadir, Morocco.

\subsection{Methods}

2.2.1. Preparation of PVA (0.5\%) Solution in Water. PVA solution was prepared, in order to be employed as stabilizer, via dissolving of $0.5 \mathrm{~g}$ of PVA in $100 \mathrm{~mL}$ flask and adequate amount of deionized water was added to make up the desired volume. PVA was dissolved under magnetic stirring at $60^{\circ} \mathrm{C}$ for 40 minutes; that result is a clear PVA solution.

2.2.2. Nanoparticles Preparation. Nanoprecipitation was selected as a method of encapsulation. Furthermore for the solubility of the active molecule (indomethacin), Argan oil was considered. The encapsulation has been done in two separate steps. Firstly for the preparation of organic phase, $200 \mathrm{mg}$ of PCL was dissolved in $25 \mathrm{~mL}$ of acetone; then it was mixed with $1 \mathrm{~g}$ of Argan oil containing $4.29 \mathrm{mg}$ of indomethacin and $100 \mathrm{mg}$ of span 20. Moreover, for the preparation of the aqueous phase $50 \mathrm{~mL}$ of water, with $6.25 \mathrm{mg}$ of PVA solution, was employed. Consecutively the prepared organic phase was added dropwise to the aqueous phase.

\section{Characterization}

3.1. Particle Size Measurements. Particle size is an important characteristic which has direct link with the stability, cellular uptake, biodistribution, and drug release. Generally, the average particle size of the formed particles via preformed polymers ranges from $250 \mathrm{~nm}$ up to $500 \mathrm{~nm}$. Moreover, the size of particle through the nanoprecipitation technique is between 150 and $500 \mathrm{~nm}$. The average particle size of the prepared indomethacin containing PCL-particles was measured via dynamic light scattering technique in $0.1 \mathrm{mM}$ $\mathrm{NaCl}$ solution at $25^{\circ} \mathrm{C}$ using Zetasizer NanoZS2000 (Malvern instrument). Each recorded value was the average of 3 measurements.

3.2. Zeta Potential Measurements. The charge of particles surfaces is obtained by the value of zeta potential which is 
related to the chemical nature of the polymer, surfactant, and $\mathrm{pH}$ of the medium [5]. The zeta potential and the surface charge density are the characteristics of the particles that can be directly associated with the stability of the colloidal system and in vivo performance of the particles [11]. Zeta potential of the prepared colloidal particles has been measured using Malvern Zetasizer (NanoZS2000) instrument. The particles (after solvent evaporation) were first diluted with $1 \mathrm{mM} \mathrm{NaCl}$ solution and then were measured at $25^{\circ} \mathrm{C}$. The reported zeta potential values are the average of 3 measurements.

3.3. Indomethacin Encapsulation Efficiency (EE) Measurements. In the nanoprecipitation technique of encapsulation there are various factors which determine the EE, such as chemical nature of active molecule and polarity of the drug. With taking into account these issues the EE of hydrophilic molecule can be maximally $10 \%$. However, EE of lipophilic drug could reach $70 \%$ [11]. The EE of indomethacin in the prepared nanoparticles has been calculated.

3.4. Transmission Electron Microscopy (TEM). The Argan oil polymer based colloidal nanoparticle morphology and microstructures were studied by TEM employing Phillips CM120 microscope, CMEABG, University of Claude Bernard Lyon I. In brief, a drop of sample that is already diluted with water was deposited onto a carbon-coated copper grid and then it was left to dry at room temperature overnight before TEM imaging. In fact, TEM analysis of nanoparticles was carried out to observe the nanoparticles formation and to know about encapsulation of Argan oil.

3.5. Stability Study of Nanoparticles Containing Indomethacin. Frequently, because of many factors such as nanoparticles composition, parameters related to the encapsulation technique, and storage condition, it is complicated to determine the stability of the nanoparticles [5]. However, here to assess the stability of the developed formulations, particle size, surface charge, and loading efficiency of nanoparticles were measured at room temperature and normal sunlight after each 10 days. For this end, the nanoparticles were prepared with $1 \mathrm{~g}$ Argan oil.

To study the effect of $\mathrm{pH}$ on the stability of the formulation, zeta potential of the nanocapsules containing indomethacin has been measured at different $\mathrm{pH}$ values of $2,5,7,9$, and 11 .

It might be concluded that $\mathrm{pH}$ change has no considerable effect on the stability of the Argan oil containing nanocapsules, which makes clear the stability of the prepared formulation by electrostatic stability.

3.6. Indomethacin Nanoparticles Preparation Reproducibility Test. To evaluate the reproducibility of this formulation, 3 preparations containing $1 \mathrm{~g}$ Argan oil including indomethacin under same operating conditions have been fabricated. Their particles size, zeta potential, and encapsulation efficiency were measured.

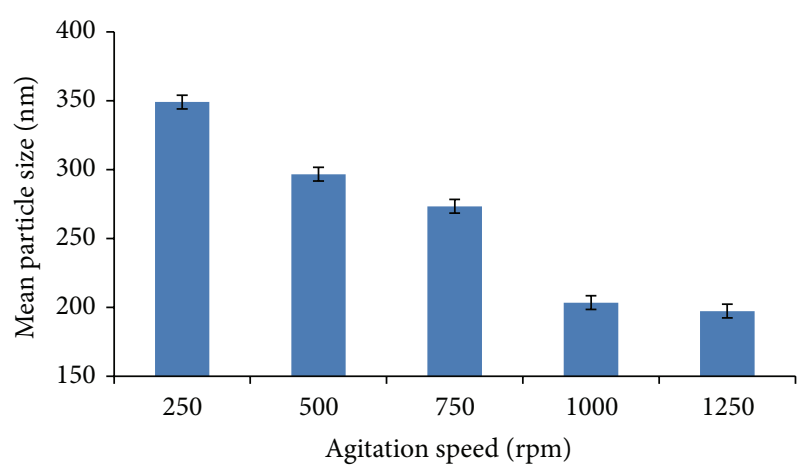

FIgURE 1: Agitation speed effect on the nanoparticles size.

\section{Results and Discussion}

The goal of this investigation is to encapsulate indomethacin in PCL based nanoparticles via nanoprecipitation method. A systematic study of nanoparticles formulation without indomethacin was performed. Different materials related parameters such as polymer (polycaprolactone) concentration, Argan oil concentration, organic/aqueous phase ratio, and nature and concentration of stabilizers were evaluated. Operating related parameters like stirring speed, organic phase addition speed, and temperature of the aqueous phase, on colloidal properties (i.e., particles size, zeta potential, size distribution, stability, and morphology), have been also studied. In second time, the encapsulation of indomethacin, its characterization, stability, and reproducibility test had been performed.

According to the results it might be possible to encapsulate $1 \mathrm{~g}$ of Argan oil containing indomethacin within polycaprolactone as a polymer. The originality of this investigation lies in the dual action of cosmetics and therapy by using a single preparation. Compared to studies of Rosset et al. and Lococo et al., polycaprolactone was used as polymer and Argan oil amount that was used was different. These advantages were also confirmed. The use of the polycaprolactone as polymer brought the prominent issues, respectively, as (1) its compatibility with a wide range of drugs enables uniform drug distribution in the formulation matrix, (2) its long-term degradation facilitates drug release up to several months, (3) it is of nontoxic nature which has been found to be cytocompatible with several body tissues, and (4) it is biodegradable [12]. Moreover, the quantity of Argan oil containing indomethacin is important as well for providing its pharmacologic effects.

\subsection{Effect of the Operating Conditions on Indomethacin Loaded Nanoparticles}

4.1.1. Effect of Stirring Speed. The different samples were prepared by various stirring speed and in similar way. Moreover, including Argan oil ( $1 \mathrm{~g}$ ) the quantities of all ingredients in formulation were constant. There was no indomethacin in these polymer based nanoparticles. The results (Figure 1) show that by increasing of the agitation speed the particles 


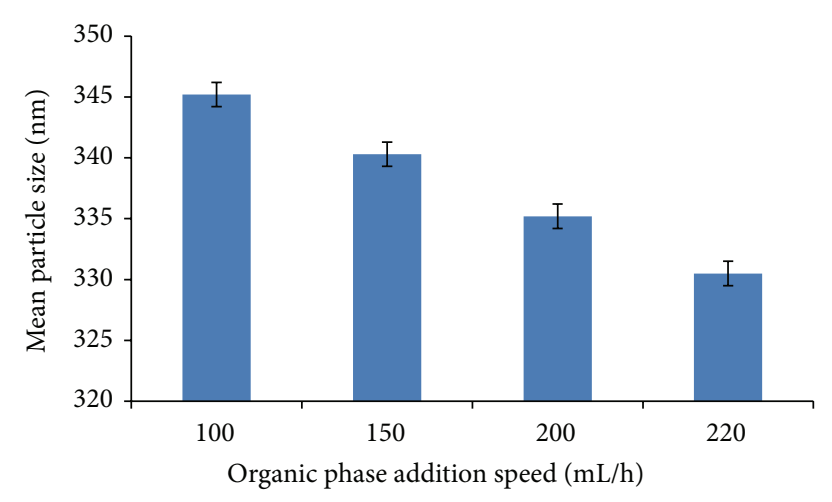

Figure 2: Effect of organic phase addition speed on particle size.

size decreased. However, in this study, the stirring speed was limited between $250 \mathrm{rpm}$ and $1250 \mathrm{rpm}$ and particle size was found that ranged from 190 to $350 \mathrm{~nm}$. It should be kept in mind that with increasing of the agitation speed up to more than $500 \mathrm{rpm}$ the polymer adheres on the walls of the beaker. The $500 \mathrm{rpm}$ was selected as optimum speed for the nanoparticles preparation.

Mora-Huertas et al. [6] have suggested that the particle formation is related to the stirring speed during organic phase addition. Therefore, additional stirring is not necessary. The high speeds of agitation break down the large drops into small drops leading to the particles [13].

4.1.2. Influence of Organic Phase Addition Speed Rate. MoraHuertas et al. [6] proposed that the organic phase addition rate can influence particle size but it depends on the organic/aqueous phase's ratio. In small volumes of organic phase, neither the organic phase addition speed nor organic phase addition manner could have considerable effect on the size of particles. Conversely, in the case of large volumes of organic phase, either the organic phase addition speed or manner might have an influence on the particle size (Figure 2).

In this study, 4 different preparations with various organic phase addition speeds of $100,150,200$, and $220 \mathrm{~mL} / \mathrm{h}$ were fabricated. The other operating condition and material related parameters are maintained unchanged. After each preparation, the particle size and the zeta potential of prepared sample have been measured. The $220 \mathrm{~mL} / \mathrm{h}$ as optimized speed for organic phase addition was selected.

According to Mora-Huertas et al. [6], while the organic phase addition speed is less the particles size will be large; the reason for this phenomenon is the difficulty in getting a homogenous mixture of phases.

4.1.3. Influence of Temperature of Organic Phase. To observe the temperature effect on the particle size and zeta potential two samples were, respectively, prepared with and without temperature. The temperature degree for heating organic phase was $40^{\circ} \mathrm{C}$. This temperature is lower than acetone boiling point $\left(56-57^{\circ} \mathrm{C}\right)$.
TABLE 1: The particle size and zeta potential of nanoparticles prepared with using and without using temperature.

\begin{tabular}{lcc}
\hline Sample & $\begin{array}{c}\text { Particle size } \\
(\mathrm{nm})\end{array}$ & $\begin{array}{c}\text { Zeta potential } \\
(\mathrm{mV})\end{array}$ \\
\hline Without temperature & $315.8 \pm 12$ & $-38.9 \pm 7$ \\
With temperature & $329.2 \pm 12$ & $-38.7 \pm 7$ \\
\hline
\end{tabular}

Table 1 shows that employing of the temperature to the aqueous phase, the size of the particles will increase as well, which is not interesting for our study because the size of nanoparticles is important for the stability and biodistribution of the nanoparticles. However, zeta potential has not changed with temperature.

\subsection{Effect of Materials from Which Formed the Polymeric Nanoparticles}

4.2.1. Effect of Polycaprolactone (PCL) Concentration on the Size of the Nanoparticles. Firstly the solubility of PCL in acetone was determined. For the determination of the maximum solubility of PCL in acetone, 10 preparations with different quantities (ranged from $100 \mathrm{mg}$ up to $1000 \mathrm{mg}$ ) of PCL, respectively, in acetone were used. After analyzing the results and investigating of the PCL solubility in acetone, it was pointed out that up to $1000 \mathrm{mg}$ PCL could be dissolved in acetone. Moreover, this study was perquisite step of the following investigation because it is important to assess organic/aqueous phase ratio effect on nanoparticle size.

The polymer concentration has a considerable importance in the size of the particles obtained by the solvent displacement technique. This method is very sensitive to alterations of polymer concentration, without considering the nature of the polymer, other operating conditions, and primary materials. Nevertheless while the polymer concentration increased the viscosity of the organic phase might be increased as well. This phenomenon may slow down solvent diffusion because of the high viscosity, which promotes the Ostwald ripening phenomenon in the emulsion, leading to increase of the particle size. Moreover, the viscosity of the organic phase is related to the polymer concentration [5].

To evaluate this parameter 5 samples with different quantities (e.g., $2 \mathrm{mg} / \mathrm{mL}, 4 \mathrm{mg} / \mathrm{mL}, 6 \mathrm{mg} / \mathrm{mL}, 8 \mathrm{mg} / \mathrm{mL}$, and $10 \mathrm{mg} / \mathrm{mL}$ ) of PCL have been prepared. As demonstrated in Figure 3 by increasing the quantity of the polymer the particle size was also found to increase.

4.2.2. Effect of the Argan Oil Presence. To evaluate the effect of Argan oil and Argan oil containing indomethacin, three preparations with $1 \mathrm{~g}$ of Argan oil and $1 \mathrm{~g}$ of Argan oil containing indomethacin and without Argan oil have been, respectively, prepared. Consecutively, the particle size and zeta potential of all 3 samples were measured. The other ingredients of formulation were kept constant.

It is reported that existence of Argan oil increases the size of nanoparticles [4]. As a confirmation of results found by Rosset et al., in fact the present work finds out that 
TABLE 2: The particle size and zeta potential of different nanoparticles prepared with different compositions.

\begin{tabular}{lcc}
\hline Sample & $\begin{array}{c}\text { Mean particle } \\
\text { size }(\mathrm{nm})\end{array}$ & $\begin{array}{c}\text { Zeta potential } \\
(\mathrm{mV})\end{array}$ \\
\hline $\begin{array}{l}\text { With 1g Argan oil } \\
\text { 1g Argan oil containing }\end{array}$ & $308 \pm 12$ & $-46 \pm 7$ \\
$\begin{array}{l}\text { indomethacin } \\
\begin{array}{l}\text { Without Argan oil and } \\
\text { indomethacin }\end{array}\end{array}$ & $321 \pm 12$ & $-40 \pm 7$ \\
\hline
\end{tabular}

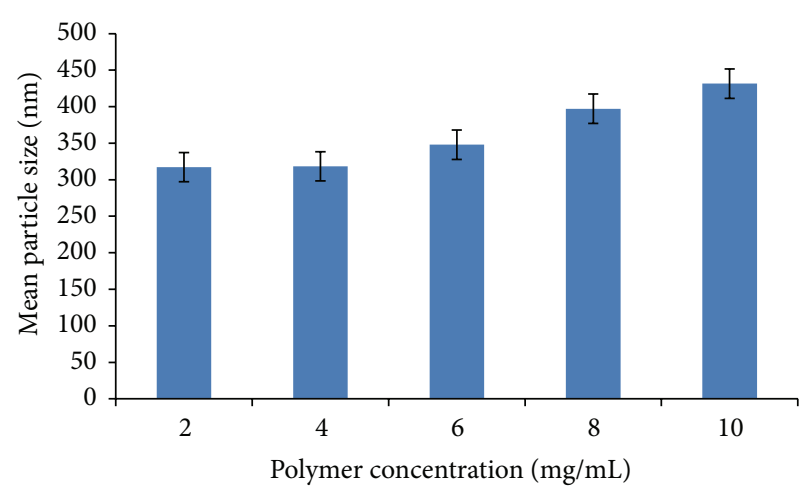

FIGURE 3: Effect of polymer concentration on the obtained particle size by solvent displacement method.

the size of particles without Argan oil and indomethacin is smaller than the size of particles which contain Argan oil and indomethacin. But in the case of zeta potential there was negative zeta potential for particles without and with Argan oil or Argan oil containing indomethacin (Table 2). Here, the zeta potential value of the particles without Argan oil and indomethacin was negative; this could be related also to the probable residual fatty acids from the synthesis of sorbitan esters (acid values of the surfactants, i.e., the quantity of free carboxylic acid groups in the surfactant) [14].

4.2.3. Effect of Argan Oil Concentration. To achieve the suitable amounts of Argan oil and polycaprolactone for nanoparticles preparation through the nanoprecipitation method, various Argan oil/polycaprolactone proportions have been studied. In this investigation range of $10 \mathrm{mg} / \mathrm{mL}$ to $50 \mathrm{mg} / \mathrm{mL}$ concentrations of Argan oil with $8 \mathrm{mg} / \mathrm{mL}$ concentration of polycaprolactone was used. The findings have shown that there is a trend to enlarging the particles size with increase of oil quantity in the formulation. The preparation through this increase of particle size might be faced with stability problems such as separation on two phases or sedimentation of large particles. According to the observations, sedimentation is due to preparation's physical stability problem. The Argan oil addition influence on zeta potential of nanoparticles can be neglected.

It was shown that $40 \mathrm{mg} / \mathrm{mL}$ of Argan oil is suitable quantity for the encapsulation or dissolution of indomethacin (Figure 4). In this investigation two hypotheses can be suggested.

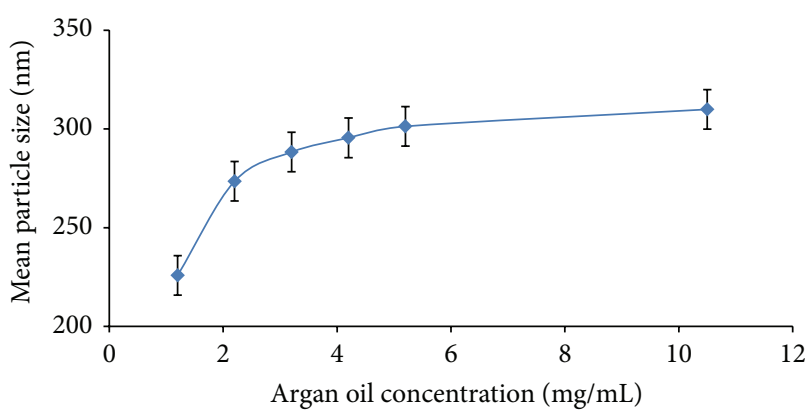

FIGURE 4: Effect of Argan oil concentration on the obtained particle size.

(i) The employed stabilizer and surfactant are useful for the stability of system to a specific quantity of Argan oil in the formulation.

(ii) Through the stable agitation speed it is difficult to disperse larger drops than optimized drops of Argan oil.

4.2.4. Effect of Stabilizers (PVA and Span 20) Nature and Concentration. Stabilizing agents are often known as key factors to maintain the stability of nanoparticles dispersion. To this end parameters such as nature and concentration of stabilizer have been investigated. In the first time, for decreasing the incompatibility problem of ingredients in formulation using minimum concentration and number of stabilizers was tried. So for this aim three preparations (with PVA, span 20 and with PVA and span 20) were made, and all the other ingredients were maintained constant. Subsequently, the particle size and zeta potential were measured.

As observed in Table 4, the size of the prepared nanoparticle was smaller while span 20 and PVA have been used together but when one of them was employed the particle size was larger.

To study the effect of PVA concentration in the formulation, 4 samples with different concentrations $(0.01,0.05$, 0.1 , and $0.5 \mathrm{mg} / \mathrm{mL}$ ) of the PVA were prepared. As pointed out in Figure 5, there was no considerable influence of different concentrations of PVA on the mean particle size. However, after a certain limit of concentration of PVA (above $0.1 \mathrm{mg} / \mathrm{mL}$ ) a large increase in particle size was observed. This sudden increase in size could be attributed to deposition of extra PVA onto the surface of particles.

To study the influence of span 20 concentration on the nanoparticles size, 5 samples with different concentration of span 20 were prepared.

Through increasing of span 20 concentration gradual increasing in size of nanoparticles size was observed as well (Figure 6).

4.2.5. Effect of Organic/Aqueous Phase Ratio. Various ratios $(0.1,0.2,0.3,0.4$, and 0.5$)$ of organic phase (acetone) and aqueous phase (water) with constant volume of water $(50 \mathrm{~mL})$ were used for the nanoparticles preparation. As seen in Figure 7, a gradual increase in mean particle size with 
TABle 3: Composition and physicochemical properties of Argan oil [14].

\begin{tabular}{|c|c|c|c|c|}
\hline $\begin{array}{l}\text { Density } \\
\left(\mathrm{g} / \mathrm{mL} ; 20 \pm 2^{\circ} \mathrm{C}\right)\end{array}$ & $\begin{array}{c}\text { Surface tension } \\
\left(\mathrm{mN} / \mathrm{m} ; 20 \pm 2^{\circ} \mathrm{C}\right)\end{array}$ & $\begin{array}{l}\text { Oil/water interfacial tension } \\
\left(\mathrm{mN} / \mathrm{m} ; 20 \pm 2^{\circ} \mathrm{C}\right)\end{array}$ & $\begin{array}{c}\text { Viscosity } \\
\left(\mathrm{mPa} \cdot \mathrm{s}, 20^{\circ} \mathrm{C}\right)\end{array}$ & Composition \\
\hline 0.91 & 23.0 & 9.8 & 65.8 & $\begin{array}{l}\text { Oleic acid (42.8\%); linoleic acid (36.8\%); palmitic } \\
\text { acid (12\%); stearic acid (6.0\%); linolenic acid } \\
(<0.5 \%) \text {. } \\
\text { Saturated acids: } 18 \% \text {; monounsaturated acids: } \\
42.8 \% \text {; diunsaturated acids: } 36.8 \%\end{array}$ \\
\hline
\end{tabular}

TABLE 4: Stabilizer role in the particle size and zeta potential.

\begin{tabular}{lcc}
\hline Sample & $\begin{array}{c}\text { Particle size } \\
(\mathrm{nm})\end{array}$ & $\begin{array}{c}\text { Zeta potential } \\
(\mathrm{mV})\end{array}$ \\
\hline With span 20 & $356 \pm 12$ & $-41 \pm 7$ \\
With PVA (0.5\% solution) & $314 \pm 12$ & $-43 \pm 7$ \\
With PVA and span 20 & $290 \pm 12$ & $-45 \pm 7$ \\
\hline
\end{tabular}

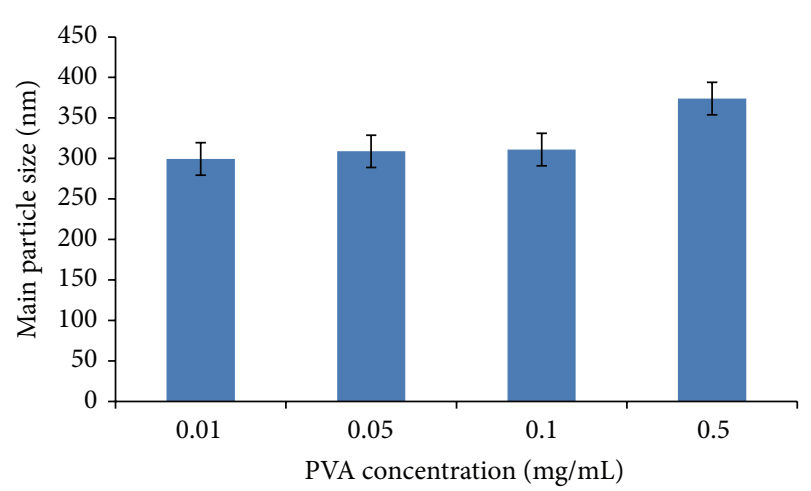

FIgURE 5: PVA concentration influence on the particle size.

an increase in organic phase was observed. The possible explanation for this increase in particle size was the further rapid diffusion of the water-miscible solvent to the aqueous phase causing quick polymer precipitation, consecutively nucleation, and, hence, the obtaining of smaller nanoparticles. These results were also in accordance with the results reported by [15]. The zeta potential of all five samples was also determined and was found to be constant at $-40 \mathrm{mV}$.

\subsection{Indomethacin Encapsulation Efficiency (EE) Measure-} ment. Here, the standard or final preparation was centrifuged. The polycaprolactone based nanoparticles settled as precipitate then were redispersed in acetonitrile and analyzed at wave length of $278 \mathrm{~nm}$. The encapsulation efficiency of the nanoprecipitation method for indomethacin was found to be $70.65 \%$. This considerable encapsulation efficiency might be due to the good solubility of indomethacin in Argan oil and also to simple and fast encapsulation technique of nanoprecipitation. From two angles this percentage of encapsulation might be interesting and in first time Argan oil is one of the oils that has not been used frequently, and it can nonetheless give good results. This is not the same case in this research. Moreover, in this work the solubility problem of indomethacin to encapsulate via nanoprecipitation technique

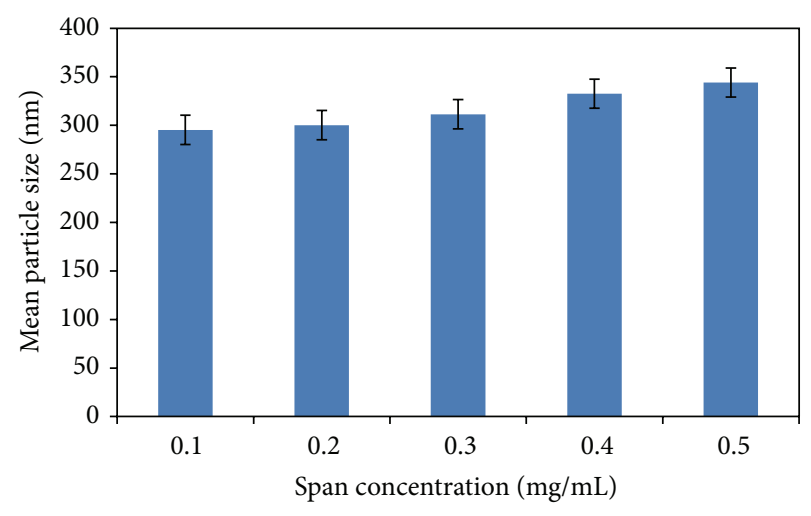

FIGURE 6: Span 20 concentration influences on the nanoparticles size.

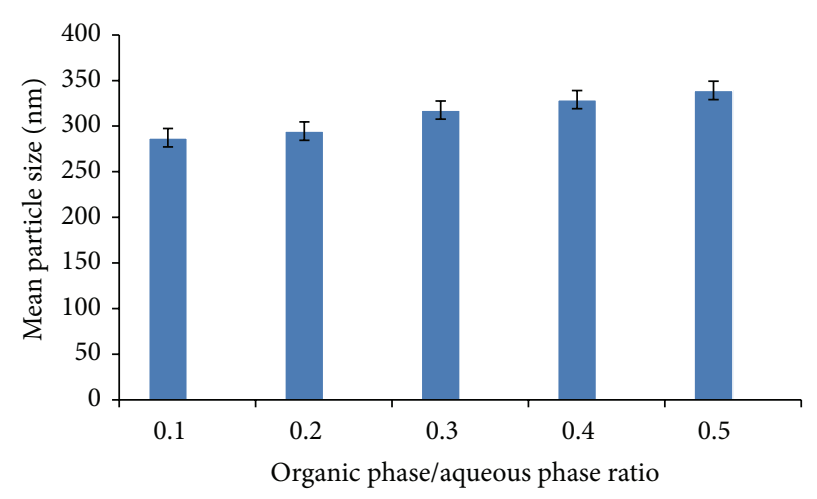

FIGURE 7: Influence of organic/aqueous phase ratio on the particle size.

by Argan oil was solved. In second time, indomethacin has excellent efficacy as anti-inflammatory, analgesic, and antipyretic agent [5]. Moreover, indomethacin according to biopharmaceutics is classified as Class II drug.

4.4. Transmission Electron Microscopy (TEM). The TEM photos (Figure 8) show that particles have two distinguished layers which confirm the encapsulation of the Argan oil through polycaprolactone. Furthermore, the center of the nanoparticle photo is less clear confirming the existence of Argan oil and the upper clear layer is the polycaprolactone.

4.5. Stability Study of Nanoparticles Containing Indomethacin. As figured out in Figure 9 after each 10-day measurement, 

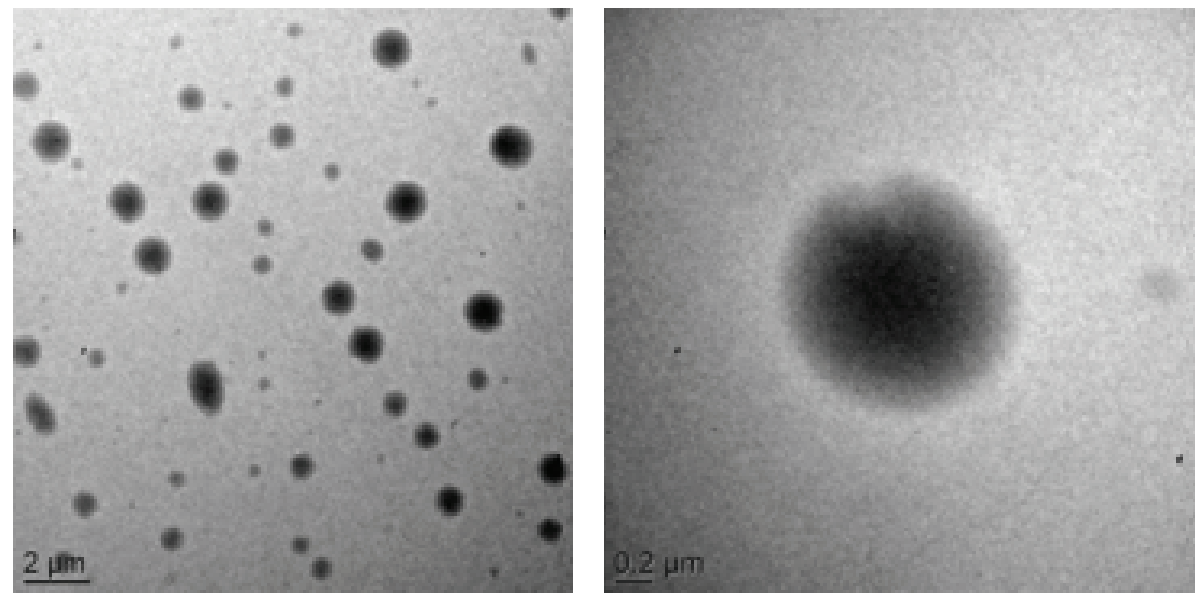

FIGURE 8: Transmission electron microscopy of polycaprolactone polymer based nanoparticles of Argan oil.

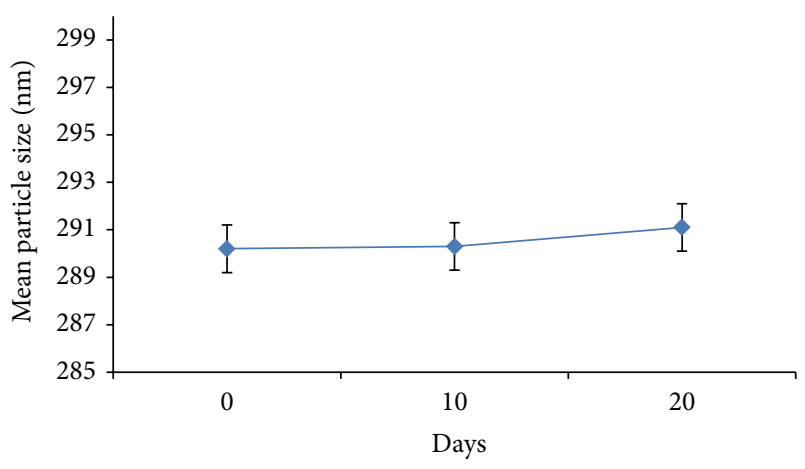

FIGURE 9: Prepared nanoparticles containing indomethacin, stability investigation after each 10 days.

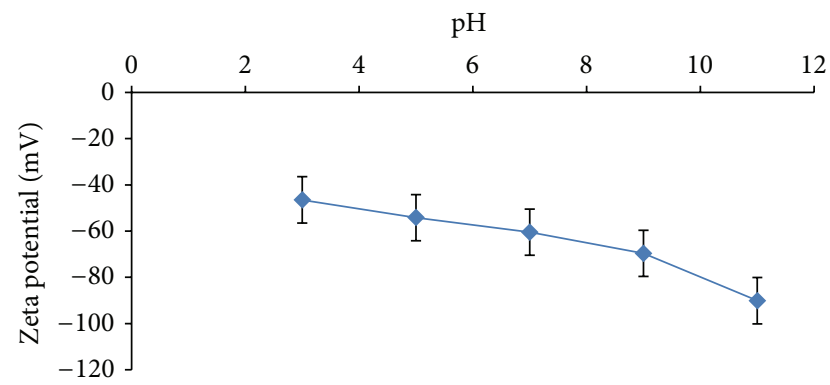

FIGURE 10: The stability of the prepared nanoparticles in different $\mathrm{pH}$.

there was no considerable change in the size and zeta potential of the nanoparticles.

The results in Figure 10 show that zeta potential values of the formulation with increasing of $\mathrm{pH}$ have become more negative. This can be attributed to the chemical nature of PCL and technique of nanoparticles preparation (nanoprecipitation).

4.6. Effect of Materials and Operating Condition Related Parameters. The charge of particles surfaces is demonstrated by the value of zeta potential which is related to the chemical nature of the polymer, surfactant, and $\mathrm{pH}$ of the medium [5]. The zeta potential and the surface charge density are the characteristics of the particles that can be directly associated with the stability of the colloidal system and in vivo performance of the particles [11].

However, to find out the effect of modification in operating condition (stirring speed, organic phase addition speed) and materials related parameters (PCL concentration, Argan oil, Argan oil/polycaprolactone ratio, PVA and span 20, and their concentration) on the zeta potential of formed nanoparticles, their zeta potential was measured after each of these alterations. It was found that these variations do not have any significant effect on the zeta potential of the dispersed system. Indeed, after measurement of the zeta potential, this value has been found between -40 and $-50 \mathrm{mV}$. These nanoparticles did not contain indomethacin. But after adding indomethacin into the nanoparticles the zeta potential was found to be the same. For the preparation of nanocapsules, when negatively charged polymers and negatively charged stabilizing agents are used, negative zeta potential values are obtained with absolute value higher than when noncharged while noncharged stabilizers are used. Furthermore, the zeta potential of particles prepared from polycaprolactone and nonionic stabilizer depended on the employed method of encapsulation [5]. Our results are consistent with those reported in the literatures. In this study the stabilizers are not changed. Then, from the obtained results it could be confirmed that the nanoparticles are stable via repulsive electrostatic interaction.

\section{Indomethacin Nanoparticles Preparation Reproducibility Test}

The results of measurement of the particles size, zeta potential, and encapsulation efficiency are represented in Table 5. From these results it could be concluded that the employed formulation with the same ingredients, quantities, and preparation conditions might give us the same results and it could be used. 
TABLE 5: Particle size, zeta potential, and encapsulation efficiency of nanoparticles containing indomethacin, prepared under the same operating condition.

\begin{tabular}{lccc}
\hline Samples & $\begin{array}{c}\text { Mean particle } \\
\text { size }(\mathrm{nm})\end{array}$ & $\begin{array}{c}\text { Zeta potential } \\
(\mathrm{mV})\end{array}$ & EE (\%) \\
\hline 1 & $349 \pm 12$ & $-43 \pm 7$ & $65 \pm 5$ \\
2 & $332 \pm 12$ & $-45 \pm 7$ & $70 \pm 5$ \\
3 & $323 \pm 12$ & $-44 \pm 7$ & $72 \pm 5$ \\
\hline
\end{tabular}

\section{Conclusion}

Delivery of different active molecules could be done through a smart approach of nanoencapsulation technology. In this investigation, polycaprolactone based nanoparticles containing indomethacin and Argan oil have been fabricated for skin application and RA treatment. The nanoparticles were prepared through the nanoprecipitation method where polycaprolactone, acetone and water, Argan oil, PVA, and span 20 are used, respectively, as polymer, solvent, carrier, and stabilizer. First, operating conditions and materials that have a considerable effect on the properties (especially particle size and zeta potential) were well studied. Moreover, systematic studies of blank nanoparticles were performed. Different materials related parameters were evaluated: polycaprolactone concentration, Argan oil presence, Argan oil concentration, organic/aqueous ratio, and nature and concentration of stabilizers. Also operation related parameters (stirring speed, organic phase addition speed, and aqueous phase temperature) influences on the colloidal properties were evaluated. In the second time, the encapsulation of indomethacin, its characterization, stability, and reproducibility test had been performed. To solve the physicochemical problem (solubility) of hydrophobic drug such as indomethacin and to attain better pharmaceutical profile of drugs, to increase the efficiency of the treatment, and to reduce the undesirable effects of NSAIDs this particulate drug delivery system could be employed. In this effort, Argan oil addition has revealed a rare direction for the application of the system towards cosmetic as well as therapeutic domains. Nanoparticles size ranging from 290 to $350 \mathrm{~nm}$ with high negative zeta potential values limited from -40 up to -50 proved the good stability of the system. An anti-inflammatory drug, indomethacin, was then successfully encapsulated and UV analysis was done to estimate the encapsulated drug amount. The encapsulation efficiency was approaching between 65 and 70\%. Above discussions allowed summarizing that nanoprecipitation is relatively simple, fast, reproducible, and low energy consuming technique to prepare polymeric nanocapsules. To complete this investigation, in vitro studies could be done. Furthermore, in vivo investigation might be performed to evaluate efficiency. Consequently, it will be more interesting to study the penetration of polycaprolactone based nanoparticles loaded with indomethacin. In addition, the penetration, delivery, toxicity, or localization of nanoparticles in skin might be investigated by the nonlocal microscope. Nanoparticles stability in accelerated and in normal condition for periods of 6 months and one year might be appealing to study as well. Encapsulation of other hydrophobic molecules by the same method and under the same condition could be possible to perform.

\section{Conflict of Interests}

We declare that we have no conflicts of interests.

\section{References}

[1] T. Delmas, A. Fraichard, P.-A. Bayle et al., "Encapsulation and release behavior from lipid nanoparticles: model study with nile red fluorophore," Journal of Colloid Science and Biotechnology, vol. 1, no. 1, pp. 16-25, 2012.

[2] A. Doustgani, E. V. Farahani, M. Imani, and A. H. Doulabi, "Dexamethasone sodium phosphate release from chitosan nanoparticles prepared by ionic gelation method," Journal of Colloid Science and Biotechnology, vol. 1, no. 1, p. 4250, 2012.

[3] C. Zandanel and C. Vauthier, "Poly (isobutylcyanoacrylate) nanoparticles decorated with Chitosan: effect of conformation of Chitosan chains at the surface on complement activation properties," Journal of Colloid Science and Biotechnology, vol. 1, no. 1, p. 6881, 2012.

[4] V. Rosset, N. Ahmed, I. Zaanoun, B. Stella, H. Fessi, and A. Elaissari, "Elaboration of argan oil nanocapsules containing naproxen for cosmetic and transdermal local application," Journal of Colloid Science and Biotechnology, vol. 1, no. 2, pp. 218-224, 2012.

[5] C. E. Mora-Huertas, H. Fessi, and A. Elaissari, "Polymerbased nanocapsules for drug delivery," International Journal of Pharmaceutics, vol. 385, no. 1-2, pp. 113-142, 2010.

[6] C. E. Mora-Huertas, H. Fessi, and A. Elaissari, "Influence of process and formulation parameters on the formation of submicron particles by solvent displacement and emulsification-diffusion methods: critical comparison," Advances in Colloid and Interface Science, vol. 163, no. 2, pp. 90-122, 2011.

[7] D. L. Scott, F. Wolfe, and T. W. J. Huizinga, "Rheumatoid arthritis," The Lancet, vol. 376, no. 9746, pp. 1094-1108, 2010.

[8] R. N. Maini, P. C. Taylor, J. Szechinski et al., "Double-blind randomized controlled clinical trial of the interleukin-6 receptor antagonist, tocilizumab, in European patients with rheumatoid arthritis who had an incomplete response to methotrexate," Arthritis and Rheumatism, vol. 54, no. 9, pp. 2817-2829, 2006.

[9] B. Tsvetkova, I. Pencheva, A. Zlatkov, and P. Peikov, "High performance liquid chromatographic assay of indomethacin and its related substances in tablet dosage forms," International Journal of Pharmacy and Pharmaceutical Sciences, vol. 4, supplement 3, pp. 549-552, 2012.

[10] M. Cherki, H. Berrougui, A. Drissi, A. Adlouni, and A. Khalil, "Argan oil: which benefits on cardiovascular diseases?" Pharmacological Research, vol. 54, no. 1, pp. 1-5, 2006.

[11] H. A. Ahad, J. Sreeramulu, C. Kumar et al., "Fabrication and in-vitro permeation studies of indomethacin-Ficus carica fruit mucilage patches," International Journal of Applied Biology and Pharmaceutical Technology, vol. 1, no. 3, pp. 786-792, 2010.

[12] T. K. Dash and V. B. Konkimalla, "Poly- $\varepsilon$-caprolactone based formulations for drug delivery and tissue engineering: a review," Journal of Controlled Release, vol. 158, no. 1, pp. 15-33, 2012.

[13] D. Ibraheem, M. Iqbal, G. Agusti, H. Fessi, and A. Elaissari, "Effects of process parameters on the colloidal properties of polycaprolactone microparticles prepared by double emulsion 
like process," Colloids and Surfaces A: Physicochemical and Engineering Aspects, vol. 445, pp. 79-91, 2014.

[14] D. Lococo, C. E. Mora-Huertas, H. Fessi, I. Zaanoun, and A. Elaissari, "Argan oil nanoemulsions as new hydrophobic drugloaded delivery system for transdermal application," Journal of Biomedical Nanotechnology, vol. 8, no. 5, pp. 843-848, 2012.

[15] M. Chorny, I. Fishbein, H. D. Danenberg, and G. Golomb, "Lipophilic drug loaded nanospheres prepared by nanoprecipitation: effect of formulation variables on size, drug recovery and release kinetics," Journal of Controlled Release, vol. 83, no. 3, pp. 389-400, 2002. 

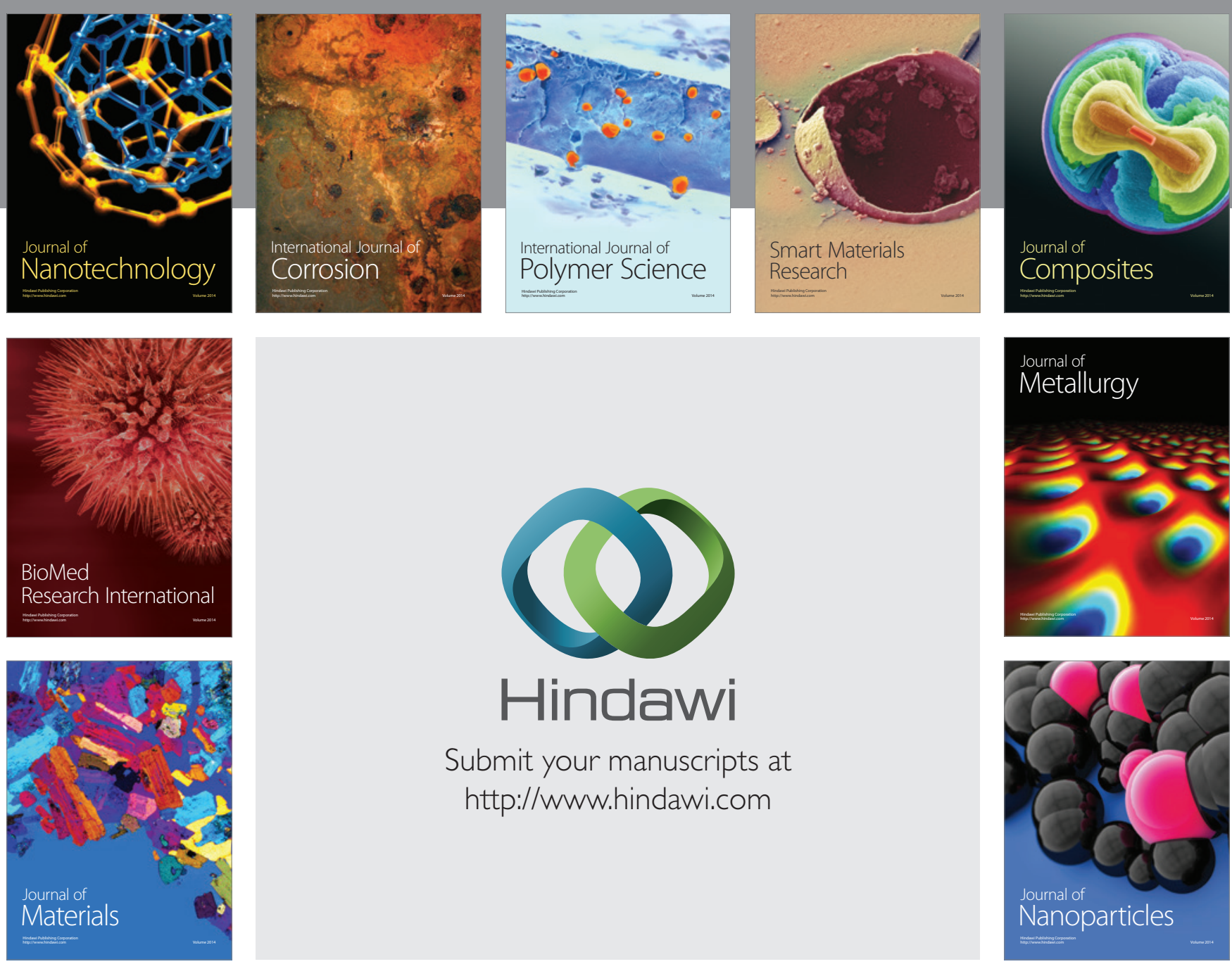

Submit your manuscripts at http://www.hindawi.com
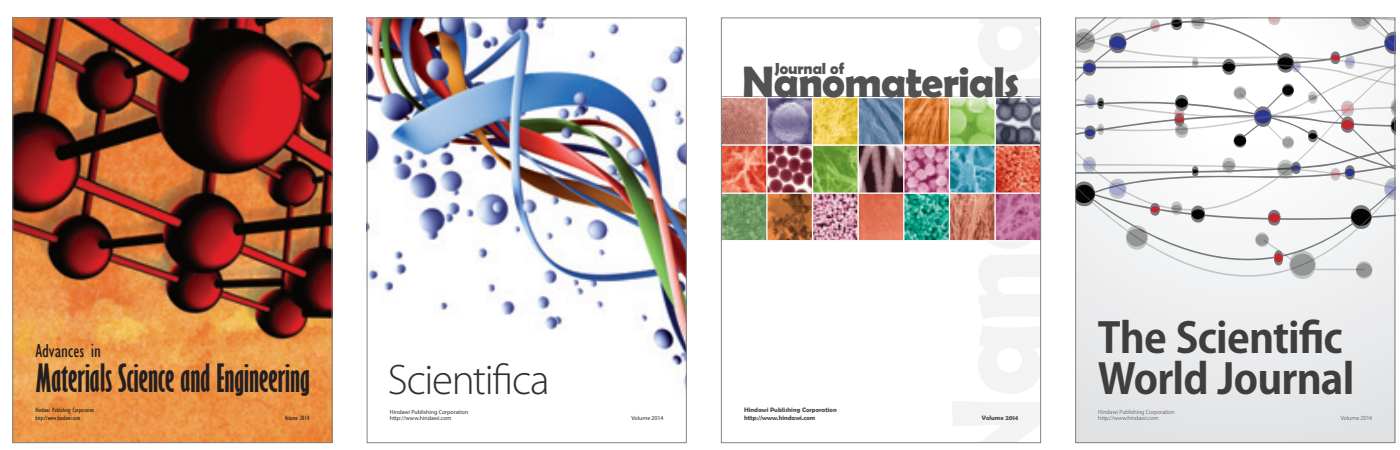

\section{The Scientific World Journal}
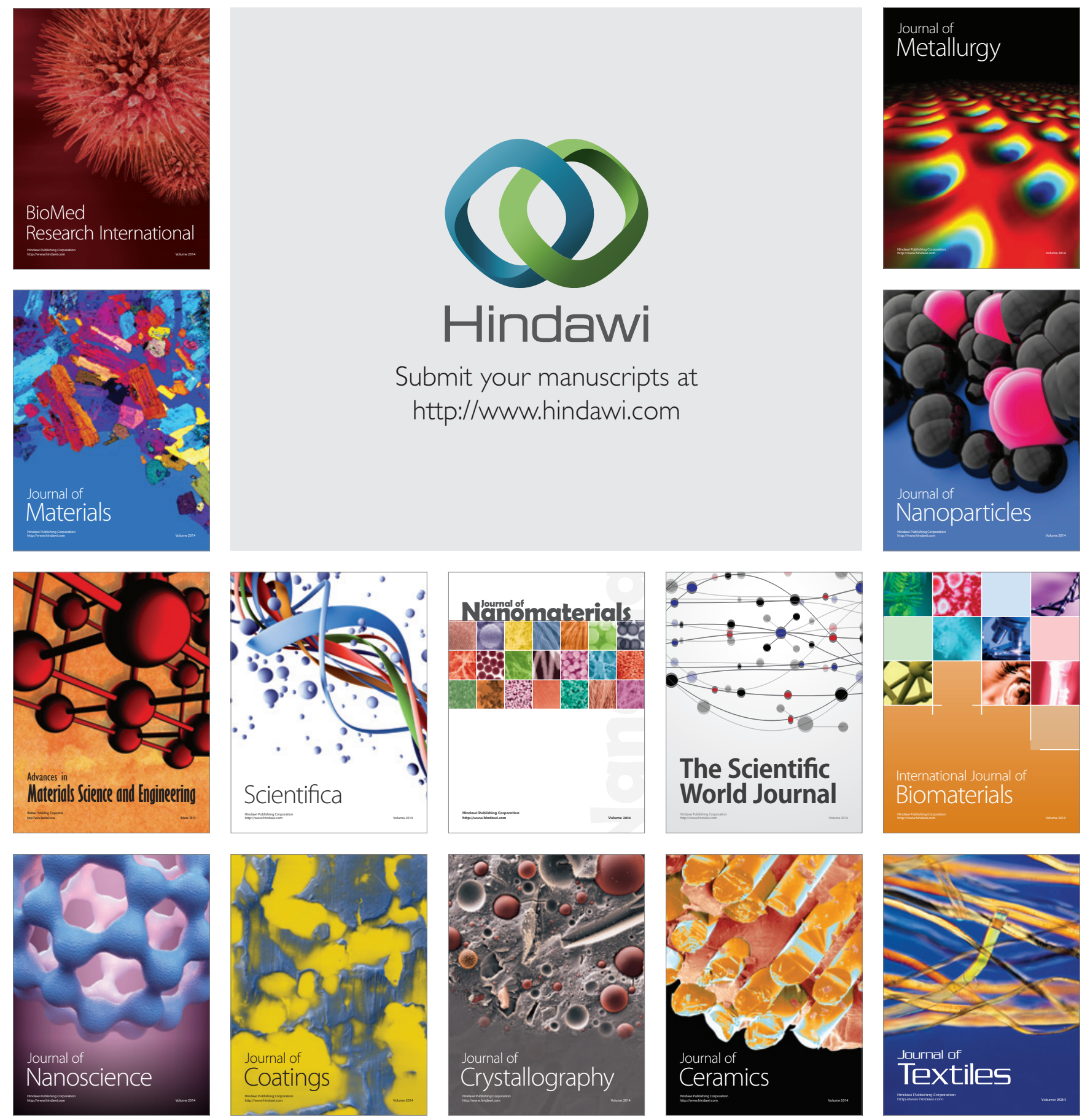